University of Wollongong

Research Online

Faculty of Engineering and Information

Faculty of Engineering and Information

Sciences - Papers: Part A

Sciences

$1-1-2013$

Rectifier capacitor filter stress analysis when subject to regular voltage fluctuations

Kun Zhao

University of Wollongong, kz664@uowmail.edu.au

Phil Ciufo

University of Wollongong, ciufo@uow.edu.au

Sarath Perera

University of Wollongong, sarath@uow.edu.au

Follow this and additional works at: https://ro.uow.edu.au/eispapers

Part of the Engineering Commons, and the Science and Technology Studies Commons

Research Online is the open access institutional repository for the University of Wollongong. For further information contact the UOW Library: research-pubs@uow.edu.au 


\title{
Rectifier capacitor filter stress analysis when subject to regular voltage fluctuations
}

\begin{abstract}
Lamp flicker levels which arise as a result of voltage fluctuations can exceed limits set by appropriate standards. New lamp types such as compact fluorescent lights (CFLs) are less sensitive to voltage fluctuations as their flicker characteristics are considerably different compared to those of the traditional incandescent lamp. These differences could support the moderation of the present voltage fluctuation and flicker standards and hence the associated limits. The potential detrimental effects on electrical equipment which may be caused by relaxation of these limits should be investigated before any changes to the present standards take place. The impact of voltage fluctuations on a fullbridge rectifier with a capacitor filter is considered as a case study in this paper. Such a circuit is found at the front end of many different types of equipment that are connected to the public AC supply network. The capacitor ripple current characteristic is of particular interest. The research reported in this paper indicates that the filter capacitor will accumulate and dissipate increased amount of charge when the rectifier is subjected to AC source voltage fluctuations. The consequence is that the RMS value of the capacitor current will increase where the magnitude of this increase is related to the modulation frequency and magnitude of the fluctuating voltage. Therefore, an AC supply with a voltage fluctuation component will cause a full-bridge rectifier with capacitor filter to sustain increased stress. This stress may accelerate the capacitor ageing process, eventually resulting in premature equipment failure. The research indicates that there is a need for indices other that the short-term and long-term flicker indices required for equipment compatibility levels.
\end{abstract}

\section{Keywords}

filter, stress, analysis, when, subject, regular, voltage, fluctuations, rectifier, capacitor

\section{Disciplines}

Engineering | Science and Technology Studies

\section{Publication Details}

K. Zhao, P. Ciufo \& S. Perera, "Rectifier capacitor filter stress analysis when subject to regular voltage fluctuations," IEEE Transactions on Power Electronics, vol. 28, (7) pp. 3627-3635, 2013. 


\title{
Rectifier Capacitor Filter Stress Analysis When Subject to Regular Voltage Fluctuations
}

\author{
Kun Zhao, Student Member, IEEE, Phil Ciufo, Senior Member, IEEE, and Sarath Perera, Member, IEEE
}

\begin{abstract}
Lamp flicker levels that arise as a result of voltage fluctuations can exceed limits set by appropriate standards. New lamp types such as compact fluorescent lights are less sensitive to voltage fluctuations as their flicker characteristics are considerably different compared to those of the traditional incandescent lamp. These differences could support the moderation of the present voltage fluctuation and flicker standards and hence the associated limits. The potential detrimental effects on electrical equipment which may be caused by relaxation of these limits should be investigated before any changes to the present standards take place. The impact of voltage fluctuations on a full-bridge rectifier with a capacitor filter is considered as a case study in this paper. Such a circuit is found at the front end of many different types of equipment that are connected to the public ac supply network. The capacitor ripple current characteristic is of particular interest. The research reported in this paper indicates that the filter capacitor will accumulate and dissipate increased amount of charge when the rectifier is subjected to ac source voltage fluctuations. The consequence is that the RMS value of the capacitor current will increase, where the magnitude of this increase is related to the modulation frequency and magnitude of the fluctuating voltage. Therefore, an ac supply with a voltage fluctuation component will cause a fullbridge rectifier with capacitor filter to sustain increased stress. This stress may accelerate the capacitor ageing process, eventually resulting in premature equipment failure. The research indicates that there is a need for indices other that the short-term and long-term flicker indices required for equipment compatibility levels.
\end{abstract}

Index Terms-Electrolytic capacitor, equivalent series resistance (ESR), flicker, rectifier current ripple, voltage fluctuation.

\section{NOMENCLATURE}

$V_{p} \quad$ Amplitude of the fundamental ac voltage.

$f_{c} \quad$ Fundamental frequency.

$f_{m} \quad$ Modulation frequency.

$m \quad$ Modulation depth.

$\Delta V \quad$ Voltage magnitude variation.

$P_{\text {st }} \quad$ Short-term flicker severity index.

$P_{\mathrm{lt}} \quad$ Long-term flicker severity index.

$T_{\text {core }} \quad$ Capacitor core temperature.

$T_{a} \quad$ Capacitor ambient temperature.

$R_{\mathrm{th}} \quad$ Capacitor thermal resistance.

$P_{\text {loss }} \quad$ Capacitor total power loss.

Manuscript received May 28, 2012; revised September 15, 2012 and August 1, 2012; accepted October 24, 2012. Date of current version December 24, 2012. Recommended for publication by Associate Editor D. Vinnikov.

The authors are with the School of Electrical, Computer and Telecommunications Engineering, and the Endeavour Energy Power Quality and Reliability Centre, University of Wollongong, Wollongong, N.S.W. 2522, Australia (e-mail: kz664@uowmail.edu.au; ppc@uow.edu.au; sarath@uow.edu.au).

Color versions of one or more of the figures in this paper are available online at http://ieeexplore.ieee.org.

Digital Object Identifier 10.1109/TPEL.2012.2228279

\author{
$R_{1 \text { base }} \quad$ Base resistance of capacitor. \\ E Temperature sensitivity factor. \\ $T_{\text {base } \quad \text { Base temperature. }}$ \\ $T \quad$ Period of capacitor current calculation. \\ $i_{c} \quad$ Capacitor instantaneous current. \\ a Slope of the trailing edge. \\ $Q_{\text {chg_a }} \quad$ Capacitor total charge with nonfluctuating ac power \\ supply. \\ $Q_{\text {dis_a }} \quad$ Capacitor total discharge with nonfluctuating ac \\ power supply. \\ $I_{\text {peak_a }} \quad$ Capacitor charging current peak value with non- \\ fluctuating ac power supply. \\ $t_{a} \quad$ Capacitor charging time in each half-cycle with \\ nonfluctuating ac power supply. \\ $I_{\text {dis }} \quad$ Capacitor discharging current. \\ $I_{c r m s \_a} \quad$ RMS value of the total capacitor current with non- \\ fluctuating ac power supply. \\ $I_{\text {chg_rms_a }}$ RMS value of the capacitor charging current with \\ nonfluctuating ac power supply. \\ $I_{\text {dis_rms_a }}$ RMS value of the capacitor discharging current \\ with nonfluctuating ac power supply. \\ $Q_{\text {chg_b }} \quad$ Capacitor total charge with a fluctuating ac power \\ supply.

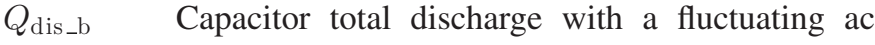 \\ power supply.

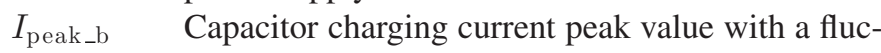 \\ tuating ac power supply. \\ $t_{b} \quad$ Capacitor charging time in each half-cycle with a \\ fluctuating ac power supply. \\ $I_{\text {crms_b }} \quad$ RMS value of the total capacitor current with a \\ fluctuating ac power supply. \\ $I_{\text {chg_rms_b }} \quad$ RMS value of the capacitor charging current with \\ a fluctuating ac power supply. \\ $I_{\text {dis_rms_b }} \quad$ RMS value of the capacitor discharging current \\ with a fluctuating ac power supply. \\ M Similar triangle coefficient.
}

\section{INTRODUCTION}

E LECTROMAGNETIC compatibility standards and guidelines [1] used in the determination of compatibility levels and network planning levels of voltage fluctuations at various points of the power system use flicker severity indices as the basis of the procedure. Voltage fluctuations are defined as repetitive or random variations in the magnitude of the supply voltage. These fluctuations may be caused by rapidly changing loads, whose power demand is not constant, or by loads which are continually connected and disconnected. If the fluctuations are severe enough, they may lead to light flicker, resulting in 
an unsteadiness of visual sensation. Therefore, flicker can be used as a metric to indicate the level of voltage fluctuation. The flicker level depends on the amplitude, frequency, and duration of voltage fluctuations.

The luminous output of the incandescent lamp is affected by voltage fluctuations. These lamps are slowly being replaced by other, higher efficiency lamp types in the residential lighting market, such as compact fluorescent and other energy saving lamps. The literature [2] shows that the luminous output of these new lamp types has a reduced sensitivity to voltage fluctuations than the conventional incandescent lamp. This property is being used to support a discussion about a relaxation of the recommended or normative flicker index limits [3].

However, the potential detrimental effects caused by voltage fluctuations on electrical equipment should be considered before the flicker limits are relaxed. Among the range of various power quality (PQ) issues, flicker is not normally considered as a serious problem for equipment. This stems from the conventional thought that electrical equipment can tolerate the voltage changes [4]. For this reason, there are few investigations carried out on electrical equipment performance and the potential effects caused by voltage fluctuations of the type that are responsible for flicker.

As an indispensable, cost-effective component, the fullbridge diode rectifier combined with a smoothing capacitor is widely applied at the input stage of switch mode power supplies (SMPS), most adjustable-speed drives (ASDs), and other types of electronic equipment. The component combination is directly connected to the public supply network. Thus, the impact on a full-bridge diode rectifier caused by supply voltage fluctuations is of primary interest.

The aluminum electrolytic capacitor is common design choice due to its large capacitance, high energy storage, and low price. Unfortunately, the electrolytic capacitor is responsible for most failures of power supplies and ASD lifetime degradation [5]-[18]. Furthermore, its short life span normally cannot meet the requirements for long-life system design. The common opinion about electrolytic capacitor lifetime degradation is that a high operating temperature will accelerate the process of electrolyte diffusion through the capacitor seals and the processes that result in chemical changes of the electrolyte and the oxide layer are accelerated at high temperature [9], [19], [20]. In addition, capacitor ripple current and ambient temperature are the main contributors to operating temperature increase. Attention in this paper is concentrated on the rectifier filter capacitor ripple current profile when the capacitor is subjected to regular sinusoidal voltage fluctuations.

This paper is organized as follows: background details in relation to voltage fluctuations and flicker as a PQ issue are reviewed in Section II. The characteristics of aluminum electrolytic capacitor are briefly described in Section III, including the power loss mechanism, thermal model, and lifetime degradation process. Section IV presents the full-bridge rectifier with filter-capacitor performance, particularly capacitor ripple-current mathematical modeling under ideal power supply conditions and regular sinusoidal voltage fluctuations (flicker) conditions. As a case study, Section V presents capacitor ripple current performance when

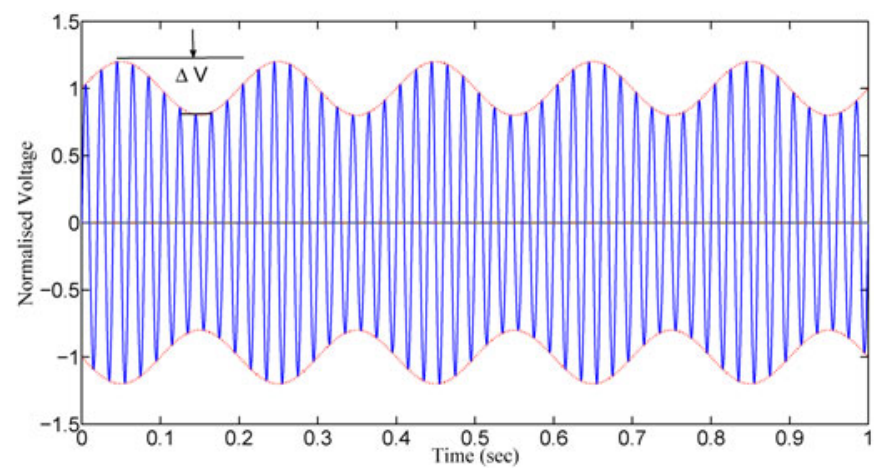

Fig. 1. Example of a regular voltage fluctuation waveform.

subjected to a constant voltage fluctuation level with various modulation frequencies, including simulation results and experimental work. Finally, conclusions are presented in Section VI.

\section{REVIEW OF Voltage FluCtUATIONS AND FLICKER}

The term flicker can be used to identify and describe the voltage fluctuation phenomenon in PQ studies. The term is derived from the impact that voltage fluctuations have on incandescent lamps such that they are perceived by the human eye to light flicker. Analysis is generally focused on flicker in the frequency range from $0.5 \mathrm{~Hz}$ to approximately $35 \mathrm{~Hz}$ since at these frequencies, flicker sensitivity is higher for the human eye-brain system, resulting in most customer complaints.

Fig. 1 illustrates a voltage fluctuation example [21] that is considered in flicker studies which arise from sinusoidal amplitude modulation (AM). The corresponding instantaneous voltage with a fluctuation component can be expressed as

$$
\begin{aligned}
v(t) & =V_{p} \sin \left(2 \pi f_{c} t\right)\left[1+m \sin \left(2 \pi f_{m} t\right)\right] \\
m & =\frac{\Delta V}{2 V_{p}}
\end{aligned}
$$

where $V_{p}$ is the amplitude of the fundamental ac voltage, $f_{c}$ is the fundamental frequency, and $f_{m}$ is the modulation frequency. Moreover, $\Delta V$ represents the voltage magnitude variation and modulation depth is expressed as $m$. The perceptibility of flicker depends on the voltage change magnitude $\Delta V$ and modulation frequency $f_{m}$. The spectral component of the voltage supply with sinusoidal AM can be established by expanding (1)

$$
\begin{aligned}
v(t)= & V_{p} \sin \left(2 \pi f_{c} t\right)+\frac{m V_{p}}{2} \sin \left(2 \pi\left(f_{c}+f_{m}\right) t-\frac{\pi}{2}\right) \\
& +\frac{m V_{p}}{2} \sin \left(2 \pi\left(f_{c}-f_{m}\right) t+\frac{\pi}{2}\right) .
\end{aligned}
$$

According to (2), the flicker voltage source contains two frequency components in addition to the fundamental frequency $f_{c}$ : one is at a supersynchronous frequency that can be considered as an upper-sideband (USB with a frequency of $f_{c}+f_{m}$ ) and another at a subsynchronous frequency that can be considered as a lower-sideband (LSB with a frequency $f_{c}-f_{m}$ ). Such spectral components can generally exist as combinations of various frequency components that are superimposed on the fundamental frequency with same magnitude and contrary phase angles. 


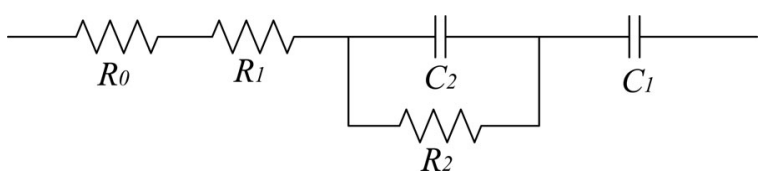

Fig. 2. Electrolytic capacitor model [5].

Combined with voltage change magnitude and modulation frequency, the severity of voltage fluctuations can be defined [21] by the short-term flicker severity index $P_{\text {st }}$ and the longterm flicker severity index $P_{l t}$. These two indices are important in determining compatibility and planning levels. For the measurement of voltage fluctuations, the flickermeter can be used to quantify flicker metrics.

\section{Aluminum EleCtrolytic CAPACITOR CHARACTERISTICS}

As documented in the literature [20], the aluminum electrolytic capacitor is the primary selection for industrial applications due to its relatively large capacity and low price. Electrolytic capacitors are mostly used in various power converters in the dc-bus circuit for smoothing voltage ripple, including full-bridge rectifiers, buck and boost converters, etc. However, this type of capacitor is responsible for most of the failures of power supplies [22] and leading to poor reliability and hence short lifetime. Therefore, the aluminum electrolytic capacitor could be considered as one of the weakest components in systems such as SMPS and ASDs. The focus in previous studies [9] has been on the methods or algorithms to determine the electrolytic capacitor equivalent series resistance (ESR) since ESR can be considered as a significant indicator of electrolytic capacitor lifetime failure prediction. For this reason, it is essential to understand electrolytic capacitor ageing process mechanism.

According to [5], if the resistance $R_{0}$ represents the resistance of the foil, tabs and terminals, while $R_{1}$ accounts for the electrolyte and $C_{1}$ is terminal capacitance, and the parallel combination of $R_{2}$ and $C_{2}$ models the dielectric resistance, then the equivalent circuit in Fig. 2 represents a simplified electrical model for aluminum electrolytic capacitors. For an electrolytic capacitor, the operating voltage is, besides the temperature, a crucial stress factor contributing to the degradation of its lifetime. The higher temperatures will accelerate the deterioration mechanism which in turn accelerates chemical changes in the oxide layer and the electrolyte, leading to leakage of electrolyte vapor through the capacitor end seal. Thus, the relationship between operating temperature, capacitor ripple-current heating loss, and ESR can be described using (3)-(5) [5]

$$
\begin{aligned}
T_{\text {core }}= & T_{a}+P_{\text {loss }} R_{\mathrm{th}} \\
P_{\text {loss }}= & \sum_{n=1}^{N} I_{f_{n}}^{2} \operatorname{ESR}\left(f_{n}\right) \\
\mathrm{ESR}= & \operatorname{Real}\left(Z_{\text {cap }}\right) \\
\operatorname{Real}\left(Z_{\text {cap }}\right)= & \frac{R_{2}}{1+(2 \pi f)^{2} C_{2}{ }^{2} R_{2}{ }^{2}} \\
& +R_{0}+R_{1 \text { base }} e^{\frac{\left(T_{\text {base }}-T_{\text {core }}\right)}{E}} .
\end{aligned}
$$

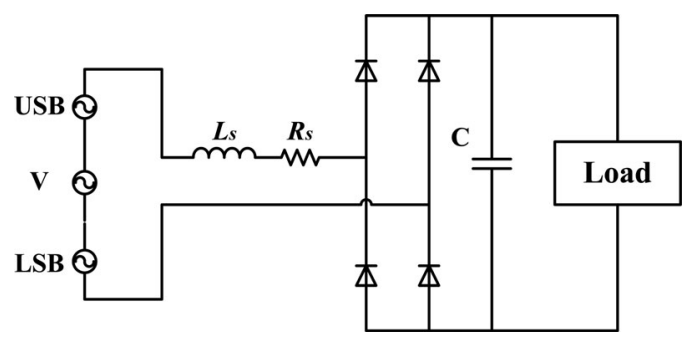

Fig. 3. Typical full-bridge rectifier.

The capacitor core temperature $T_{\text {core }}$ depends on the capacitor ambient temperature $T_{a}$, capacitor thermal resistance $R_{\mathrm{th}}$, and capacitor total power loss $P_{\text {loss }}$. Meanwhile, the nature of the electrolytic resistance $R_{1}$ is modeled with a base resistance $R_{1 \text { base }}$ and an exponential temperature variation controlled by a temperature sensitivity factor $E$. $T_{\text {base }}$ is the temperature at which the ESR was measured and $T_{\text {core }}$ is the temperature of interest for the ESR calculation.

The capacitor ripple current $I_{f_{n}}$ corresponding to relative $\operatorname{ESR}\left(f_{n}\right)$ values at frequency $f_{n}$ (where $n$ is the harmonic order) determines capacitor total power loss. Furthermore, the capacitor ESR value can be obtained by calculating the real part of the capacitor complex impedance $\operatorname{Real}\left(Z_{\text {cap }}\right)$. The capacitor ripple current and ambient temperature are the primary contributing factors for operating core temperature increase. In other words, electrolytic capacitor failure has a strong relationship with capacitor ripple current and operating ambient temperature.

\section{EVALUATION OF Full-BRIDGe RectifieR With CAPACITOR FILTER}

The circuit of a typical full-bridge rectifier with capacitor filter, which is connected between the load and power supply network, is shown in Fig. 3. Generally, the power supply includes some source impedance, comprising a series equivalent inductance $L_{s}$ and a series resistance $R_{s}$. As is well known, the filter capacitor stores energy during charging time and supplies the load during discharging time. The switching converter connected to the dc-bus side of a full-bridge rectifier is assumed to function as a constant current load [23] for the purpose of analysis.

In order to compare rectifier capacitor ripple current characteristics between ideal power supply conditions and voltage fluctuation conditions through mathematical analysis, a series of assumptions is established.

1) Capacitor charging current waveforms are considered as similar, right-angled triangles in each half-cycle, as illustrated in Figs. 4 and 5. This means that the slope of the trailing edge (hypotenuse) of all charging current waveforms is the same, expressed as $a$.

2) The capacitor discharging current (the current drawn the load connected to the dc bus) is considered as a constant current and specified as $1 \mathrm{~A}$.

3) The diodes are assumed to be ideal. The power source impedance, including the series equivalent inductance and resistance (i.e., $L_{s}$ and $R_{s}$ ) are neglected. 


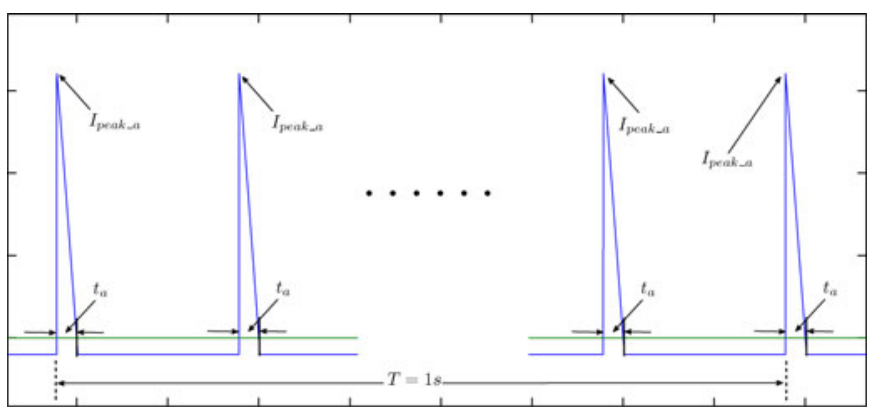

Fig. 4. Capacitor current under ideal power supply conditions.

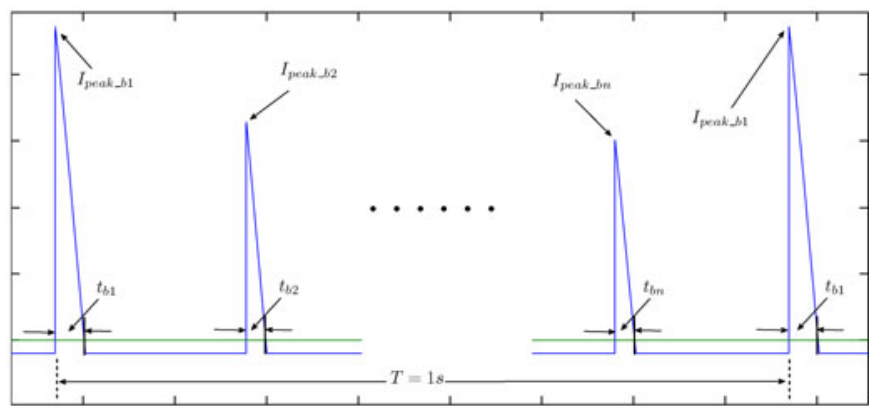

Fig. 5. Capacitor current under fluctuating voltage condition.

\section{A. Capacitor Current Analysis With the Nonfluctuating AC Power Supply}

Fig. 4 illustrates a typical rectifier capacitor-current waveform under normal power supply conditions. It shows that the capacitor charging-current peak values are constant in each ac supply half-cycle, labeled $I_{\text {peak_a }}$. In general, the RMS value of the capacitor current is calculated over a period $T\left(T=n / 2 f_{c}\right.$, where $n$ is an integer) and $f_{c}$ is the fundamental frequency of the ac supply. The reason for the use of $T$ instead of $1 / 2 f_{c}$ for the calculation of RMS value of the capacitor current will become evident in considering the case where the input voltage fluctuates, a case presented in the preceding section. Over a time period $T$, the total charge $Q_{\text {chg_a }}$ accumulated by the capacitor is the same as that is discharged $Q_{\text {dis_a }}$. This process can be described using the following equations where the capacitor instantaneous current is $i_{c}$ :

$$
\frac{1}{T} \int_{0}^{T} i_{c} d t=0
$$

such that

$$
Q_{\text {chg_a }}=Q_{\text {dis_a }}
$$

and

$$
\left\{\begin{array}{l}
Q_{\text {chg_a }}=\frac{n I_{\text {peak_a }} t_{a}}{2} \\
Q_{\text {dis_a }}=I_{\text {dis }}\left(T-n t_{a}\right)
\end{array}\right.
$$

where $t_{a}$ is the capacitor charging time in each half-cycle (as illustrated in Fig. 4) and total charging time can be expressed as $n t_{a} . I_{\mathrm{dis}}$ is the capacitor discharging current (assumed to be constant).
The RMS value of the total capacitor current $I_{\text {crms_a }}$ can be obtained using the RMS values of the capacitor charging current $I_{\text {chg_rms_a }}$ and discharging current $I_{\text {dis_rms_a }}$ as follows:

$$
\begin{aligned}
& I_{c r m s \_a}^{2}=I_{\text {chg_rms_a }}^{2}+I_{\text {dis_rms_a }}^{2} \\
& I_{\text {chg_rms_a }}^{2}=\frac{1}{T} \int_{0}^{t_{a}} n(a t)^{2} d t=\frac{n}{3 T}\left(I_{\text {peak_a }}^{2} t_{a}\right) \\
& I_{\text {dis_rms_a }}^{2}=\frac{1}{T} \int_{0}^{T-n t_{a}} I_{\text {dis }}^{2} d t=\frac{I_{\text {dis }}^{2}}{T}\left(T-n t_{a}\right) \\
& I_{\text {crms_a }}^{2}=\frac{n}{3 T}\left(I_{\text {peak_a }}^{2} t_{a}\right)+\frac{I_{\text {dis }}^{2}}{T}\left(T-n t_{a}\right)
\end{aligned}
$$

or

$$
I_{c \text { rms_a }}=\sqrt{\frac{n}{3 T}\left(I_{\text {peak_a }}^{2} t_{a}\right)+\frac{I_{\text {dis }}^{2}}{T}\left(T-n t_{a}\right)} .
$$

By substituting $T=n / 2 f_{c}$ into (13), the RMS value of the total capacitor current can be established as

$$
I_{c \mathrm{rms} \_\mathrm{a}}=\sqrt{\frac{2 f_{c}}{3}\left(I_{\text {peak_a }}^{2} t_{a}\right)+I_{\mathrm{dis}}^{2}\left(1-2 f_{c} t_{a}\right)} .
$$

\section{B. Capacitor Current Analysis With a Fluctuating AC Power Supply}

Fig. 5 illustrates the typical capacitor ripple current profile when the circuit is supplied with a fluctuating ac voltage source [24]. As expected, the instantaneous dc-bus voltage level, the ac input voltage and the load connected to the dc bus determines the capacitor ripple current behavior. The fluctuating ac source leads to dc-bus voltage fluctuations and causes the capacitor ripple current waveform to be different in each half-cycle, as illustrated in Fig. 5. It is possible that in some ac input cycles, the capacitor charging does not take place at all. On the other hand, the filter capacitor can also draw more current than normal if the ac voltage peak value is significantly greater than the dcbus voltage during some half-cycles.

Although the periodic nature of the dc bus voltage variations and the capacitor charging current waveforms are governed by the ac supply modulating frequency, for the calculation of the RMS value of the capacitor charging current, a total period of $T$ is used which is identical to that used when the ac supply was nonfluctuating. Assuming a minimum ac supply modulating frequency of $1 \mathrm{~Hz}$ (within the range $1-35 \mathrm{~Hz}$ ), T can be taken as $1 \mathrm{~s}$ for calculating the capacitor RMS current. Furthermore, over the same cycle time of $T$, the total charge which is absorbed and released by the capacitor under ideal power supply conditions is less than that under voltage fluctuation conditions (the proof is presented in the Appendix). This means that the total capacitor discharge time will increase and the corresponding charging time will decrease when considering a constant current load. Therefore, the following relationship can be given:

$$
\left\{\begin{array}{l}
Q_{\text {chg_b }}>Q_{\text {chg_a }} \\
Q_{\text {dis_b }}>Q_{\text {dis_a }}
\end{array}\right.
$$


and

$$
\left\{\begin{array}{l}
Q_{\text {chg_b }}=\frac{\sum_{i=1}^{n} I_{\text {peak_bi }} t_{b i}}{2} \\
Q_{\text {dis_b }}=I_{\text {dis }}\left(T-\sum_{i=1}^{n} t_{b i}\right)
\end{array}\right.
$$

where $Q_{\text {chg_b }}$ and $Q_{\text {dis_b }}$ represent the charge absorbed and released over the period $T$, respectively, under voltage fluctuating conditions, $I_{\text {peak } \_b n}$ stands for the capacitor peak value in each half-cycle charging time $\left(t_{b n}\right)$, as illustrated in Fig. 5.

Alternatively, (16) can be expressed as

$$
\left\{\begin{array}{l}
\frac{\sum_{i=1}^{n} I_{\text {peak } \_b i} t_{b i}}{2}>\frac{n I_{\text {peak_a }} t_{a}}{2} \\
I_{\text {dis }}\left(T-\sum_{i=1}^{n} t_{b i}\right)>I_{\text {dis }}\left(T-n t_{a}\right) .
\end{array}\right.
$$

Based on assumption 1 and the characteristics of similar triangles, the relationship between $I_{\text {peak_a }}, t_{a}$ and $I_{\text {peak_bn }}, t_{b n}$ can be described as

$$
\frac{I_{\text {peak_bi }}}{I_{\text {peak_a }}}=\frac{t_{b i}}{t_{a}}=M_{i}(i=1,2, \ldots, n)
$$

or

$$
\begin{aligned}
I_{\text {peak } \_b i} & =M_{i} I_{\text {peak } \_ \text {a }} \\
t_{b i} & =M_{i} t_{a}(i=1,2, \ldots, n)
\end{aligned}
$$

where $M_{i}$ is defined as the "similar triangle coefficient." By substituting (19) into (17), a further relationship can be established as

$$
\left\{\begin{array}{l}
\sum_{i=1}^{n} M_{i}^{2}>n \\
T-\sum_{i=1}^{n} t_{b i}>T-n t_{a} .
\end{array}\right.
$$

Obviously

$$
\sum_{i=1}^{n} M_{i}^{3}>n
$$

In addition, the RMS value of the capacitor current $I_{\text {crms_b }}$ can be established using the RMS values of charging current $I_{\text {chg_rms_b }}$ and discharging current $I_{\text {dis_rms_b }}$

$$
\begin{aligned}
I_{c \mathrm{rms} \_\mathrm{b}}^{2} & =I_{\text {chg_rms_b }}^{2}+I_{\text {dis_rms_b }}^{2} \\
I_{\text {chg_rms_b }}^{2} & =\frac{1}{T} \sum_{i=1}^{n} \int_{0}^{t_{b i}}(a t)^{2} d t \\
& =\frac{\sum_{i=1}^{n} M_{i}^{3}}{3 T}\left(I_{\text {peak_a }}^{2} t_{a}\right) \\
I_{\text {dis_rms_b }}^{2} & =\frac{1}{T} \int_{0}^{T_{b}-\sum_{i=1}^{n} t_{b i}} I_{\text {dis }}^{2} d t \\
& =\frac{I_{\text {dis }}^{2}}{T}\left(T-\sum_{i=1}^{n} t_{b i}\right)
\end{aligned}
$$

$$
\begin{aligned}
I_{c \mathrm{rms} \_\mathrm{b}}^{2}= & \frac{\sum_{i=1}^{n} M_{i}^{3}}{3 T}\left(I_{\text {peak_a }}^{2} t_{a}\right) \\
& +\frac{I_{\text {dis }}^{2}}{T}\left(T-\sum_{i=1}^{n} t_{b i}\right) .
\end{aligned}
$$

Alternatively, (25) can be expressed as

$$
I_{\text {crms_b }}=\sqrt{\frac{\sum_{i=1}^{n} M_{i}^{3}}{3 T}\left(I_{\text {peak_a }}^{2} t_{a}\right)+\frac{I_{\text {dis }}^{2}}{T}\left(T-\sum_{i=1}^{n} t_{b i}\right)} .
$$

By substituting $T=n / 2 f_{c}$ into (26)

$I_{c \text { rms_b }}=\sqrt{\frac{\sum_{i=1}^{n} M_{i}^{3} 2 f_{c}}{3 n}\left(I_{\text {peak_a }}^{2} t_{a}\right)+\frac{I_{\text {dis }}^{2}}{T}\left(T-\sum_{i=1}^{n} t_{b i}\right)}$.

By substituting (20) and (21) into (27), and comparing the result with the capacitor RMS current equation under ideal power supply conditions (14), the following relationship can be derived:

$$
I_{c \text { rms_b }}>I_{\text {crms_a }} \text {. }
$$

The preceding analysis indicates that the total quantity which is charged/discharged by the capacitor under ideal power supply conditions is less than that under voltage fluctuation conditions. The analysis also shows that the capacitor's total discharging time will increase if considering the load current as constant. This indicates that the capacitor's total charging time will decrease. On the other hand, the capacitor discharging RMS current is proportional to the total discharging time. For this reason, the capacitor discharging RMS current will increase under voltage fluctuations. Additionally, for the capacitor charging RMS current, the capacitor will accumulate more charge in less time when subject to voltage fluctuations, leading to capacitor charging RMS current values increasing heavily compared to the discharging RMS current values and dominating the total capacitor RMS current increase. This conclusion will be verified in the following case study.

\section{Case Study}

As an illustrative example, a $230-\mathrm{V}$ power supply with a voltage change rate of $10 \%$ and various modulation frequencies $f_{m}$ $(1-35 \mathrm{~Hz})$ supplies a full-bridge rectifier with a $470-\mu \mathrm{F}$ capacitor and a $300-\Omega$ load, as illustrated in Fig. 6 . The fluctuating voltage source can be considered as an ideal source superimposed by positive and negative sequence voltages with frequency $f_{m}$ (modulation frequency). This leads to two sideband frequency components; $f_{c}+f_{m}$ and $f_{c}-f_{m}$. This balanced sideband frequency component can pass through the rectifier and appear in the capacitor current spectrum. The simulation and experimental results showing capacitor current characteristics, including charging/discharging performance and current frequency spectrum, are summarized in Figs. 7-12.

Fig. 7 displays the capacitor ripple current frequency spectrum under modulation frequencies in the range of $10-1000 \mathrm{~Hz}$. It is clear from the figure that the modulation frequency $(10 \mathrm{~Hz})$ component appears as USB and LSB in the capacitor current 


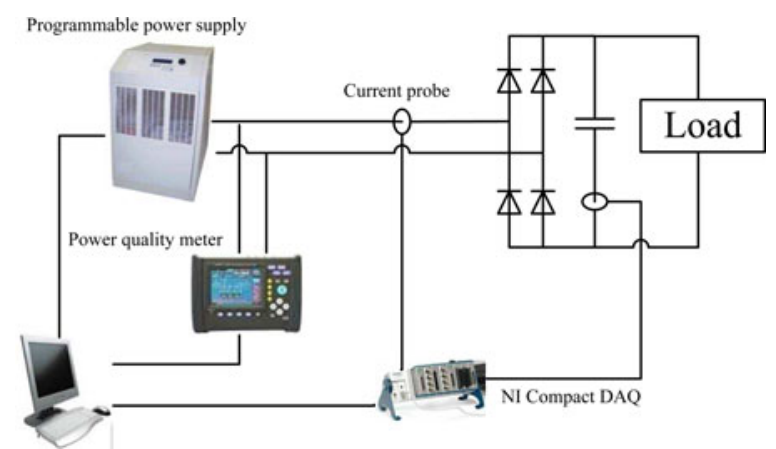

Fig. 6. Experiment schematic diagram.

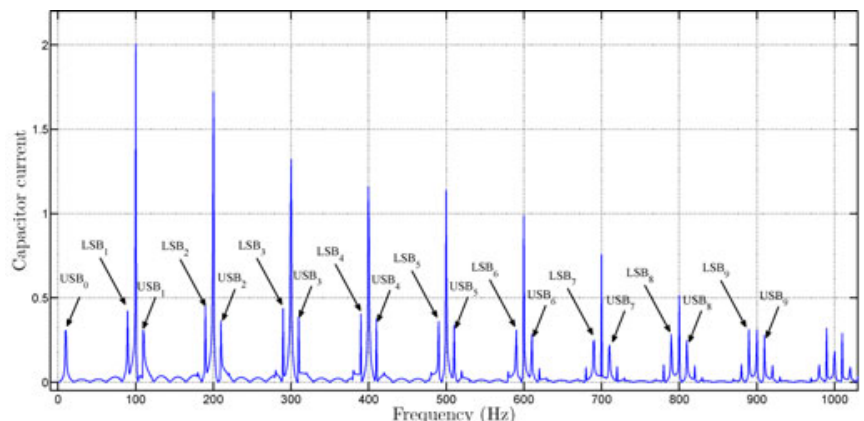

(a)

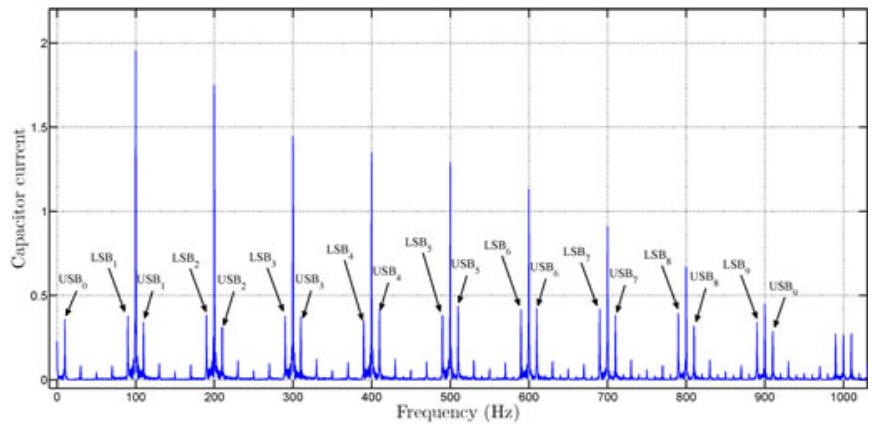

(b)

Fig. 7. Capacitor current frequency spectrum with $f_{m}=10 \mathrm{~Hz}$. (a) Simulation results. (b) Experimental results.

frequency spectrum, which is centered around the capacitor current harmonic frequencies (i.e., $100 \mathrm{~Hz}, 200 \mathrm{~Hz}, \ldots$... In addition, Fig. 7 also shows that the LSB magnitude of capacitor current harmonics is not equal to the USB, both in simulation and experimental results. Furthermore, in the higher frequency range, the current sidebands are even higher than their "family" harmonics values, as the tenth harmonic $(1000 \mathrm{~Hz})$ and its sideband shows. Some smaller sideband harmonics also appear which are clearly observed in the experiment analysis.

Fig. 8 illustrates the simulation (blue line) and experimental (red line) results of the capacitor total charging and discharging characteristics during a period $T(T=10 \mathrm{~s})$ with a modulation frequency ranging from 1 to $35 \mathrm{~Hz}$. In Fig. 8(b), both the simulation and experimental results show that the capacitor total discharge time keeps increasing as the modulation frequency increases and is proportional to the capacitor charge accumulation/dissipation quantities. On the other hand, the reader should note that the capacitor total charging time decreases as

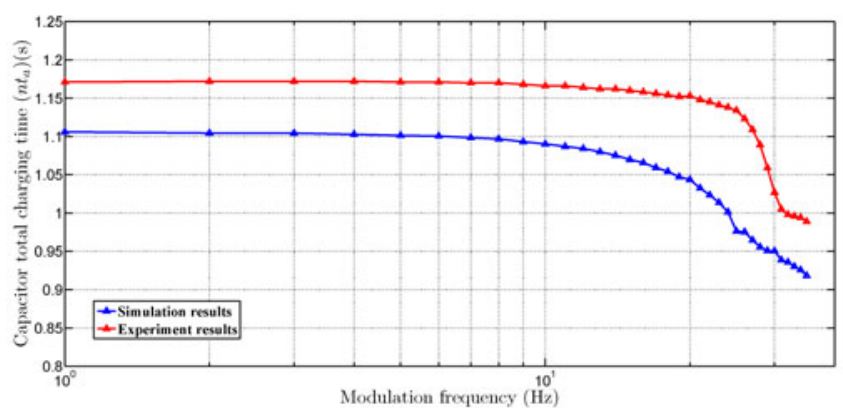

(a)

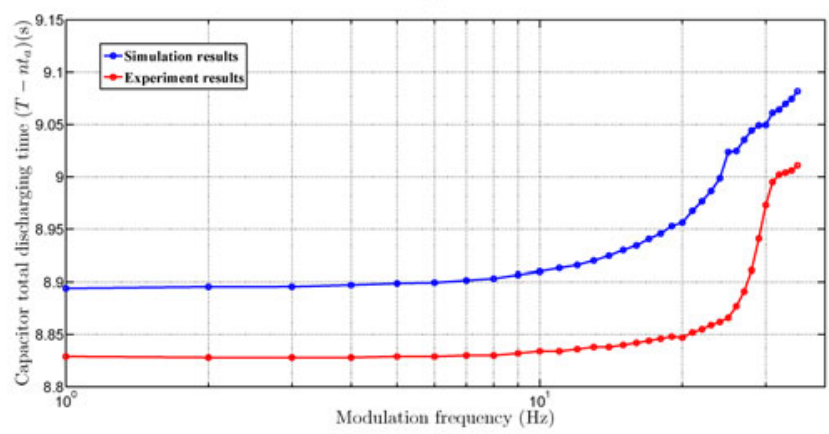

(b)

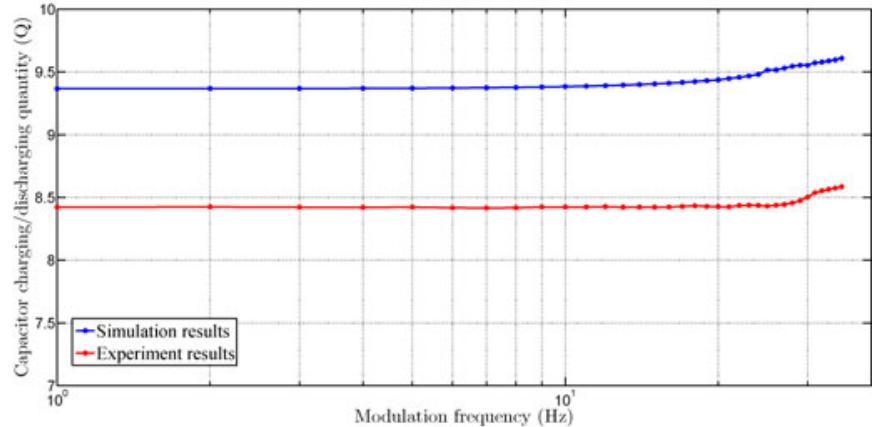

(c)

Fig. 8. Variation of capacitor charge/discharge behavior with modulation frequency. (a) Capacitor total charging time. (b) Capacitor total discharging time. (c) Capacitor charging/discharging quantity.

the modulation frequency increases. This means that the capacitor will accumulate more charge in less time, particularly at higher modulation frequencies which verifies the analysis in previous section. Furthermore, in the comparison of simulation and experimental results which is illustrated in Fig. 8, the reader should note that the capacitor charging time, discharging time and charge/discharge rates all change dramatically at modulation frequencies beyond $20 \mathrm{~Hz}$. Thus, the higher modulation frequency should be given more attention according to capacitor charging/discharge behavior.

Figs. 9 and 10 display the RMS value of different capacitor current components in relation to increasing modulation frequencies. The figures show that, as modulation frequency increases, capacitor RMS current increases dramatically, particularly at higher modulation frequencies. Capacitor RMS charging current and RMS discharging current are the two contributors to the total RMS value, as shown in the mathematical analysis in Section IV. Both simulation and experimental results indicate that the RMS discharging current increases slowly in relation to 


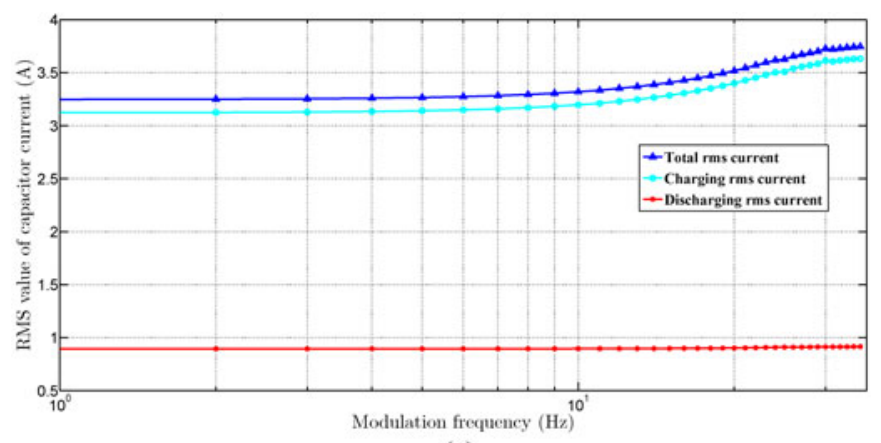

(a)

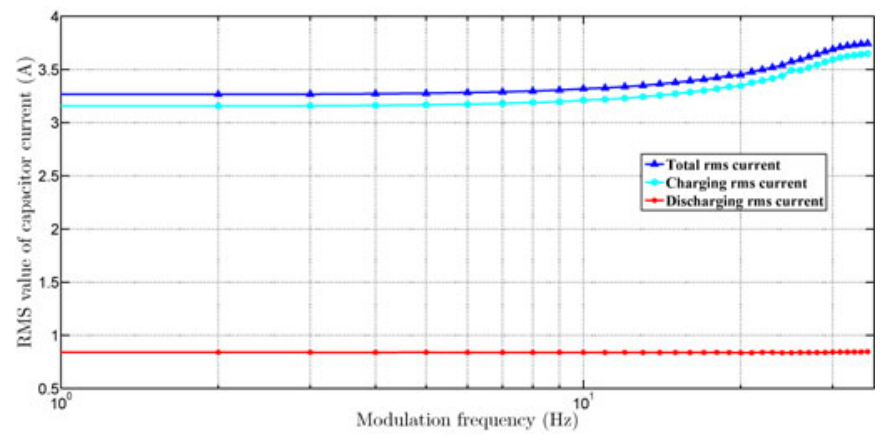

(b)

Fig. 9. Variation of capacitor current RMS value with modulation frequency. (a) Simulation results. (b) Experiment results.

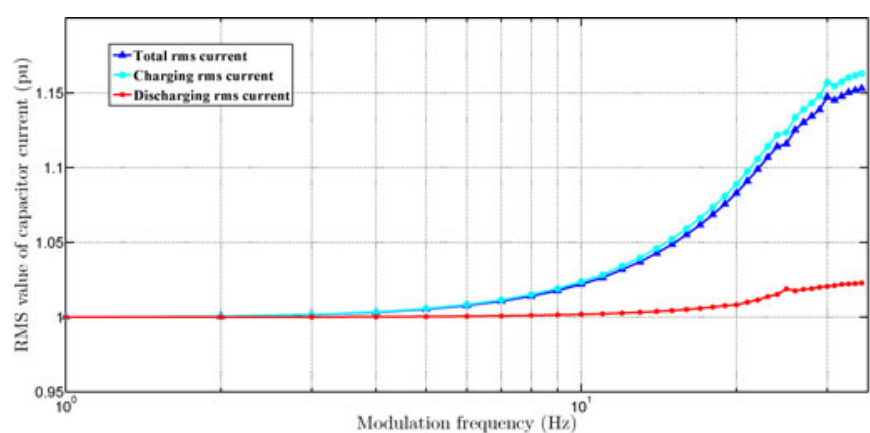

(a)

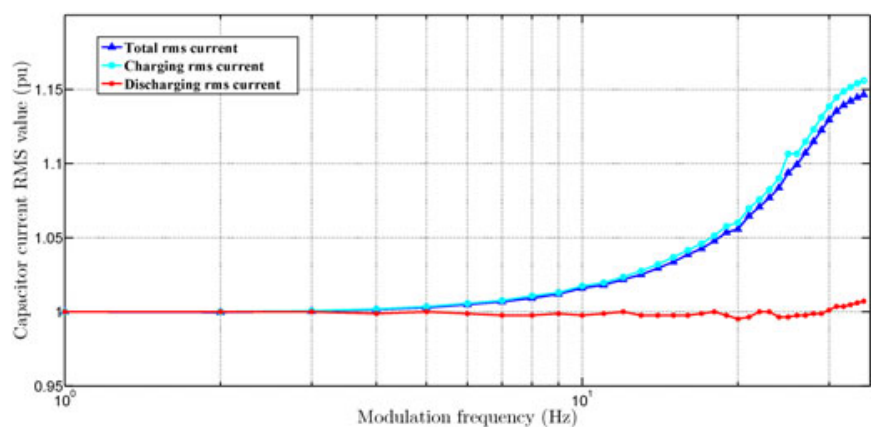

(b)

Fig. 10. RMS value of capacitor current variation with modulation frequency in per unit. (a) Simulation results. (b) Experiment results.

an increasing modulation frequency. The primary reason is that the capacitor dissipates more charge during the longer discharge time that occurs under frequency modulation conditions.

Compared to the discharging current, the RMS charging current value increases dramatically with increasing modulation

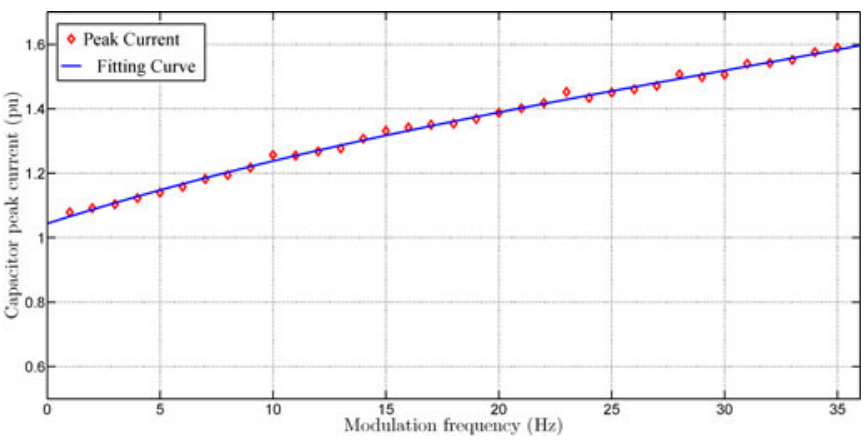

Fig. 11. Variation of capacitor peak current profile at $\frac{\Delta V}{V}=10 \%$ with modulation frequency.

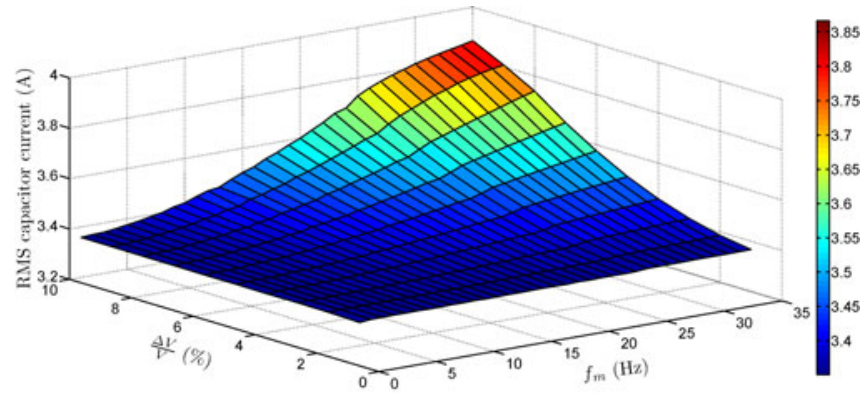

(a)

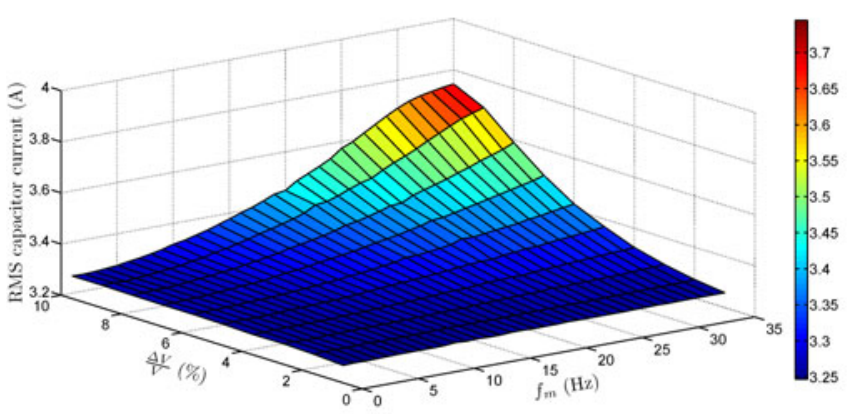

(b)

Fig. 12. Variation of capacitor RMS current with $f_{m}$ and $\frac{\Delta V}{V}$. (a) Simulation results. (b) Experiment results.

frequencies, especially at modulation frequencies over $20 \mathrm{~Hz}$. Under such conditions, the capacitor accumulates more charge and the total charging time is reduced, which results in the capacitor charging current waveform becoming more distorted than under ideal conditions. This situation is worse with an increasing modulation frequency. The RMS value (p.u.) in Fig. 10 reveals that the charging current RMS value increases by up to approximately $116.3 \%$ (115.6\% for experimental result) of its value under ideal power supply conditions with a $35 \mathrm{~Hz}$ modulation frequency. Meanwhile, the total RMS current value increases by up to approximately $115.3 \%$ (114.6\% for experimental result).

However, the discharging current RMS values almost remains the same as the modulation frequency increases. Therefore, one can summarize that compared to capacitor discharging current, the RMS charging current dominates the total capacitor current RMS value. Since the capacitor RMS current is a crucial factor which accelerates capacitor degradation and ageing time (especially aluminum electrolytic capacitor), the capacitor RMS 


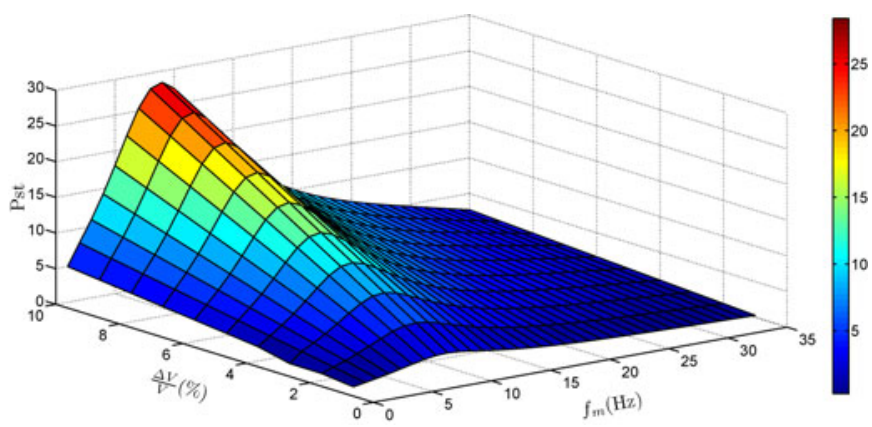

Fig. 13. Variation of $P_{\mathrm{st}}$ with $f_{m}$ and $\frac{\Delta V}{V}$.

current behavior, particularly the RMS charging current component, should be given more attention under high modulation frequency conditions.

Additionally, Fig. 11 displays the capacitor peak current profile with $10 \%$ voltage fluctuation conditions. Fig. 11 illustrates that the capacitor charging current peak value is higher than under normal conditions. The situation worsens when the modulation frequency is $35 \mathrm{~Hz}$; the value is higher by about $60 \%$. This means that the capacitor charging peak value will increase the risk of capacitor dielectric breakdown.

By investigating the rectifier capacitor current performance with voltage change rates from $1 \%$ to $10 \%$ and modulation frequencies from $1 \mathrm{~Hz}$ to $35 \mathrm{~Hz}$, the capacitor RMS current characteristics can be summarized in Fig. 12. Fig. 12 illustrates the change in capacitor RMS current trend with different voltage change rates and modulation frequencies. The surface shows that the voltage change $\Delta V$ and modulation frequency $f_{m}$ are the main reasons behind the capacitor RMS current increase. Comparing the two surfaces, the experimental results are in good agreement with the simulation work. Fig. 12 indicates that rectifier capacitor RMS current increases quickly under higher voltage change rates and higher modulation frequencies. Therefore, a higher voltage change and higher modulation frequency will increase the rectifier capacitor breakdown risk.

However, this risk situation cannot be represented by using the flicker level index $P_{\text {st }}$ since $P_{\mathrm{st}}$ is derived from the impact perceived by the human eye to light flicker. The $P_{\mathrm{st}}$ level can be illustrated in Fig. 13 with various voltage change rates and modulation frequencies. According to the $P_{\text {st }}$ curve, the peak value arises at approximately $8 \mathrm{~Hz}$ and decreases as the modulation frequency increases. Comparing Figs. 12 and 13, there is a higher rectifier capacitor current but lower flicker level under high voltage change and high modulation frequency conditions. Thus, $P_{\mathrm{st}}$ is not appropriate for determining power system electromagnetic compatibility levels and planning levels by only applying $P_{\text {st }}$ values. In addition, relaxation of allowable flicker levels will raise the potential detrimental risk for rectifier capacitors.

\section{CONCLUSION}

An investigation has been carried out to examine the singlephase rectifier filter capacitor stress when subjected to regular voltage fluctuations. The analysis indicates that the filter capacitor used in the dc side of a rectifier circuit will accumulate and dissipate more charge when it is subjected to input voltage fluctuations. The consequence is that the RMS value of the capacitor current will increase. Meanwhile, a simulation and a related experiment shows that the capacitor charging time decreases dramatically and RMS current increases quickly when the modulation frequency is beyond about $20 \mathrm{~Hz}$. In addition, sidebands, which are centered around the capacitor current harmonic frequencies, appear.

For electrolytic capacitors, the ripple current is one important factor which affects the ageing process. Voltage fluctuations result in the capacitor RMS current increasing dramatically and this phenomenon keeps deteriorating as the voltage change rates and fluctuation frequency increases. These ripple currents have the undesirable effects of raising the electrolytic capacitor temperature and accelerating their ageing process.

Both simulation and experimental work indicate that higher voltage change rates and modulation frequencies should be considered very carefully. Therefore, a new or advanced voltage fluctuation index needs to be proposed in order to indicate the risk to equipment since $P_{\mathrm{st}}$ does not sufficiently reflect this risk.

\section{APPENDIX A}

\section{Capacitor Charge Analysis With Fluctuating AC POWER SUPPLY}

In order to compare over the same cycle time, the total charge that is absorbed and released by the capacitor under ideal power supply condition with that under voltage fluctuation conditions, three possibilities can be given

1)

$$
\left\{\begin{array}{l}
Q_{\text {chg_b }}<Q_{\text {chg_a }} \\
Q_{\text {dis_b }}<Q_{\text {dis_a }} .
\end{array}\right.
$$

By substituting (19) into (29) and simplifying

$$
\left\{\begin{array}{l}
\sum_{i=1}^{n} M_{i}^{2}<n \\
\sum_{i=1}^{n} M_{i}>n .
\end{array}\right.
$$

As is well known, $M_{i}$ is positive real number and $n$ is integer. Thus, (30) is contradiction.

2)

$$
\left\{\begin{array}{l}
Q_{\text {chg_b }}=Q_{\text {chg_a }} \\
Q_{\text {dis_b }}=Q_{\text {dis_a }} .
\end{array}\right.
$$

By substituting (19) into (31) and simplifying

$$
\left\{\begin{array}{l}
\sum_{i=1}^{n} M_{i}^{2}=n \\
\sum_{i=1}^{n} M_{i}=n
\end{array}\right.
$$

According to Lagrange multiplier minima rules, if (32) is correct, the only condition is that $M_{i}$ is the same value. This means that the capacitor charge current waveform 
is same in each half-cycle (no voltage fluctuation condition). Obviously, this is not true for voltage fluctuation conditions. Therefore, the only conclusion is that over the same cycle time $T$, the total charge which is absorbed and released by the capacitor under ideal power supply condition is less than that under voltage fluctuations condition. This can be explained by the following equations:

3)

$$
\left\{\begin{array}{l}
Q_{\text {chg_b }}>Q_{\text {chg_a }} \\
Q_{\text {dis_b }}>Q_{\text {dis_a }} .
\end{array}\right.
$$

By substituting (19) into (33) and simplifying

$$
\left\{\begin{array}{l}
\sum_{i=1}^{n} M_{i}^{2}>n \\
\sum_{i=1}^{n} M_{i}>n .
\end{array}\right.
$$

Mathematically, (34) is correct when taking into account that $M_{i}$ is a positive real number and $n$ is an integer.

\section{REFERENCES}

[1] Electromagnetic Compatibility (EMC)-Part 3-7: Limits - Assessment of Emission Limits for the Connection of Fluctuating Installations to MV, HV and EHV Power Systems, IEC 61000-3-7. Edition 2.0 (2008-02) Std.

[2] R. Cai, J. Cobben, J. Myrzik, J. Blom, and W. Kling, "Flicker responses of different lamp types," IET Generation, Transmiss. Distrib., vol. 3, no. 9, pp. 816-824, Sep. 2009.

[3] C. Medeiros and J. de Oliveira, "Effects of voltage fluctuation associated to flicker limits on equipments performance," in Proc. 10th Int. Conf. Harmonics, Quality, Power, vol. 1, Oct. 2002, pp. 347-352.

[4] R. Redl, "Electromagnetic environmental impact of power electronics equipment," Proc. IEEE, vol. 89, no. 6, pp. 926-938, Jun. 2001.

[5] M. Gasperi, "Life prediction modeling of bus capacitors in ac variablefrequency drives," IEEE Trans. Ind. Appl., vol. 41, no. 6, pp. 1430-1435, Nov./Dec. 2005.

[6] D. C. Lee, K. J. Lee, J. K. Seok, and J. W. Choi, "Online capacitance estimation of dc-link electrolytic capacitors for three-phase ac-dc-ac PWM converters using recursive least squares method," IEE Proc.-Electr. Power Appl., vol. 152, no. 6, pp. 1503-1508, Nov. 2005.

[7] K. Lee, T. Jahns, G. Venkataramanan, and W. Berkopec, "DC-bus electrolytic capacitor stress in adjustable-speed drives under input voltage unbalance and sag conditions," IEEE Trans. Ind. Appl., vol. 43, no. 2, pp. 495-504, Mar./Apr. 2007.

[8] A. Amaral and A. Cardoso, "An economic offline technique for estimating the equivalent circuit of aluminum electrolytic capacitors," IEEE Trans. Instrum. Meas., vol. 57, no. 12, pp. 2697-2710, Dec. 2008.

[9] Y. M. Chen, H. C. Wu, M. W. Chou, and K. Y. Lee, "Online failure prediction of the electrolytic capacitor for LC filter of switching-mode power converters," IEEE Trans. Ind. Electron., vol. 55, no. 1, pp. 400-406, Jan. 2008.

[10] K. W. Lee, M. Kim, J. Yoon, S. B. Lee, and J. Y. Yoo, "Condition monitoring of dc-link electrolytic capacitors in adjustable-speed drives," IEEE Trans. Ind. Appl., vol. 44, no. 5, pp. 1606-1613, Sep./Oct. 2008.

[11] K. Tsang and W. Chan, "Low-cost sensing of equivalent series resistance for electrolytic capacitors," IET Power Electron., vol. 2, no. 5, pp. 555562, Sep. 2009.

[12] A. Amaral and A. Cardoso, "A simple offline technique for evaluating the condition of aluminum-electrolytic-capacitors," IEEE Trans. Ind. Electron., vol. 56, no. 8, pp. 3230-3237, Aug. 2009.

[13] K. Abdennadher, P. Venet, G. Rojat, J.-M. Re andtif, and C. Rosset, "A real-time predictive-maintenance system of aluminum electrolytic capacitors used in uninterrupted power supplies," IEEE Trans. Ind. Appl., vol. 46, no. 4, pp. 1644-1652, Jul./Aug. 2010.

[14] A. Braham, A. Lahyani, P. Venet, and N. Rejeb, "Recent developments in fault detection and power loss estimation of electrolytic capacitors," IEEE Trans. Power Electron., vol. 25, no. 1, pp. 33-43, Jan. 2010.
[15] M. Vogelsberger, T. Wiesinger, and H. Ertl, "Life-cycle monitoring and voltage-managing unit for dc-link electrolytic capacitors in PWM converters," IEEE Trans. Power Electron., vol. 26, no. 2, pp. 493-503, Feb. 2011.

[16] A. Amaral and A. Cardoso, "On-line fault detection of aluminium electrolytic capacitors, in step-down dc-dc converters, using input current and output voltage ripple," IET Power Electron., vol. 5, no. 3, pp. 315-322, Mar. 2012.

[17] H. Gualous, H. Louahlia, and R. Gallay, "Supercapacitor characterization and thermal modelling with reversible and irreversible heat effect," IEEE Trans. Power Electron., vol. 26, no. 11, pp. 3402-3409, Nov. 2011.

[18] W. Chen and S. Hui, "Elimination of an electrolytic capacitor in ac/dc lightemitting diode (LED) driver with high input power factor and constant output current," IEEE Trans. Power Electron., vol. 27, no. 3, pp. 15981607, Mar. 2012.

[19] R. Alwitt and R. Hills, "The chemistry of failure of aluminum electrolytic capacitors," IEEE Trans. Parts, Mater. Packag., vol. PMP-1, no. 2, pp. 28 34, Sep. 1965.

[20] K. Lee, T. Jahns, T. Lipo, G. Venkataramanan, and W. Berkopec, "Impact of input voltage sag and unbalance on dc-link inductor and capacitor stress in adjustable-speed drives," IEEE Trans. Ind. Appl., vol. 44, no. 6, pp. 1825-1833, Nov./Dec. 2008.

[21] Electromagnetic Compatibility (EMC)-Part 4-15: Testing and Measurement Techniques - Flickermeter - Function and Design Specifications, IEC 61000-4-15. Edition 2.0 (2010-08) Std.

[22] A. Lahyani, P. Venet, G. Grellet, and P. J. Viverge, "Failure prediction of electrolytic capacitors during operation of a switchmode power supply," IEEE Trans. Power Electron., vol. 13, no. 6, pp. 1199-1207, Nov. 1998.

[23] J. Doval Gandoy, C. Castro, and M. Martinez, "Line input ac-to-dc conversion and filter capacitor design," IEEE Trans. Ind. Appl., vol. 39, no. 4, pp. 1169-1176, Jul./Aug. 2003.

[24] K. Zhao, P. Ciufo, and S. Perera, "Lifetime analysis of aluminum electrolytic capacitor subject to voltage fluctuations," in Proc. 14th Int. Conf. Harmonics, Quality, Power, Sep. 2010, pp. 1-5.

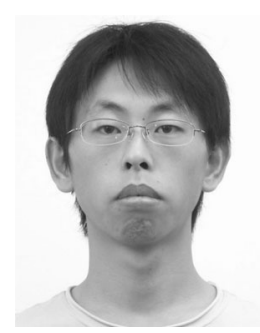

Kun Zhao (S'10) received the B.S. degree in electrical and electronics engineering from Wuhan University, Wuhan, China, in 2004, and the M.Eng.Sc. degree from the University of Wollongong, Wollongong, N.S.W., Australia, in 2009, where he is currently working toward the Ph.D. degree in electrical engineering.

His research interests include power quality and the effect of power quality on equipment operation and lifetime.

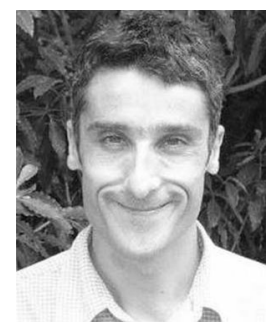

Phil Ciufo (SM'07) received the B.E. degree (Hons.) in electrical engineering in 1990, while also completing an Industry Cadetship, the M.E. degree (Hons.) in electrical engineering in 1993, and the Ph.D. degree in 2002, all from the University of Wollongong, Wollongong, N.S.W., Australia.

He joined the academic staff of the university after completing his Ph.D. He has had various stints in industry as an Electrical Engineer and returned to academia in 2007. His research interests include modeling and analysis of power distribution systems, advanced distribution automation, and power quality.

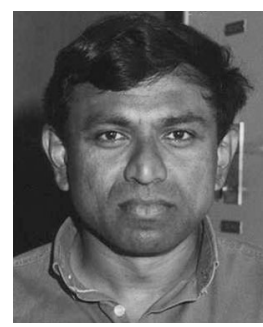

Sarath Perera (M'95) received the B.Sc.(Eng.) degree in electrical power engineering from the University of Moratuwa, Moratuwa, Sri Lanka, the M.Eng.Sc. degree from the University of New South Wales, Sydney, N.S.W., Australia, and the $\mathrm{Ph} . \mathrm{D}$. degree from the University of Wollongong, Wollongong, N.S.W.

He was a Lecturer at the University of Moratuwa. $\mathrm{He}$ is currently an Associate Professor with the University of Wollongong. He is the Technical Director of the Endeavour Energy Power Quality and Reliability Centre at the University of Wollongong. 